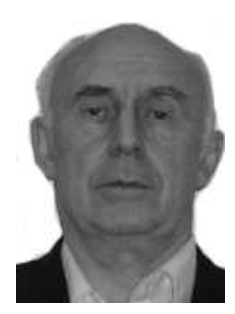

ДМИТРИЕВ

Борис Михайлович (МГТУ им. Н.Э. Баумана)

DMITRIEV

Boris Mikhailovich

(Moscow, Russian Federation, Bauman Moscow State Technical University)
Причины изменения свойств конструкции станка под действием термических явлений

\section{Б.М. Дмитриев}

В соответствии с современными тенденциями в сфере производства изделий машиностроения станочные системы характеризуются высокой энерговооруженностью, концентрацией производимых действий по изготовлению деталей на одном станке, а также высокими скоростями резания, большими значениями подач и т. д. Станки оборудованы разнообразными устройствами, обеспечивающими автоматизацию вспомогательных процессов. Все перечисленное и многое другое во время функиионирования сопровождается теплообразованием, вследствие которого конструкиия, подвергаясь действию тепла, изменяет жесткость несущей системы.

Для снижения уровня воздействия необходимо знать первопричину изменения жесткости. Первые работы в этом направлении констатировали факт наличия данного явления. Позднее была установлена причина изменения жесткости в шпиндельных опорах и выявлен механизм процесса изменения жесткости в опорах шпинделей. Однако это не может оббяснить механизма изменения жесткости конструкции в иелом.

В данной работе сделана попытка объяснения процесса изменения жесткости. Установлено, что изменение жесткости обусловлено движением в конструкции, возникающим под действием тепловых воздействий.

Исследование показало, что стабилизация геометрической точности конструкции является необходимым условием, но не достаточным для обеспечения точности станка. Для стабилизации жесткости конструкции станка требуется стабилизация состояния его подвижных сопряжений и неподвижных стыков по всей конструкции.

Ключевые слова: станок, термические явления, геометрическая точность, жесткость конструкции, движение конструкции, несущая система станка, изменение свойств конструкции.

\section{Causes for Changing the Construction Properties of a Machine-Tool under Heat Exposure}

\section{B.M. Dmitriev}

According to the current trends in the field of mechanical engineering the machine-tools are characterized by high available power, by concentration of actions for the manufacture of workpieces on one machine-tool, and also by high cutting speeds, high feed rates, etc. Machine-tools equipped with various devices that provide automated support processes. During the operation all of the above is accompanied by heat due to which the construction, which subjected to heat 
exposure, changes the stiffness of load-carrying system. To reduce the level of exposure it is necessary to know the root cause for stiffness changing. The first works in this field stated the presence of the phenomenon. Later the cause for stiffness changing and mechanism of the process for said changing in the spindle bearings were defined. However, this cannot explain the mechanism for stiffness changing in overall. This paper attempts to explain the process for stiffness changing. It is found that the stiffness changing is caused by the motion in construction due to heat exposure. The study showed that the stabilization of geometric accuracy of construction is essential, but not sufficient to ensure the accuracy of the machine-tool. For stiffness stabilization of the machine-tool construction it is required to stabilize the state of its moving and fixed joints throughout the construction.

Keywords:machine-tool, heat exposure, geometric accuracy, stiffness, motion of the construction, load-carrying system, changing of the construction properties.

По вопросам стабилизации геометрической точности конструкции от термических воздействий накоплен значительный опыт, как расчетный, так и экспериментальный. Разработаны мощные методы расчета, позволяющие получать существенные результаты по подвижным сопряжениям с натягом и неподвижным стыкам с натягом.

Механизм действия тепла на изменение жесткости в подвижных сопряжениях типа опоры шпинделей, представленный в работе [1], описывает процессы, которые не проявляются по внешним признакам. Основой этого механизма является процесс расширение твердого тела, в качестве которого в данном случае выступает тело шпинделя. Однако, как показывают исследования точности обработки, одним изменением жесткости шпинделя объяснить изменение жесткости всей несущей системы станка (HCC) невозможно. Для того чтобы выяснить степень влияния изменения жесткости шпинделя в общей структуре жесткости НСС был проведен эксперимент, состоящий из двух частей. В первой части установлен закон изменения жесткости НСС токарного станка, работающего на холостом ходу в течение 4 ч. При этом периодически производили измерение жесткости. Вторая часть целиком повторяла первую, но с отключенным шпинелем. Результаты исследования представлены на рис. 1.

Из полученных результатов следует, что в изменение жесткости участвует не только шпиндель, но и НСС. Закон изменения жесткости не противоречит экспоненциальной зависимости. Несущая система станка состоит, как из подвижных сопряжений, так и из неподвижных стыков, механизм изменения жесткости которых на основе расширения тела детали не поддается объяснению.

Для установления причинно-следственной связи между изменением жесткости конструкции и тепловыми воздействиями были проведены экспериментальные исследования, основой которых явились известные положения. В частности тот факт, что изменение жесткости происходит под действием теплового движения элементов конструкции.

Под действием тепловых возмущений происходит изменение положения базы под заготовку относительно базы под инструмент меняется положение баз в пространстве и времени, что по существу является признаком механического движения под действием термических сил. Любое механическое движение, в том числе и движение конструкции под действием теплоты, однозначно описывается двумя параметрами: законом и траекторией движения. Для определения количественных показателей

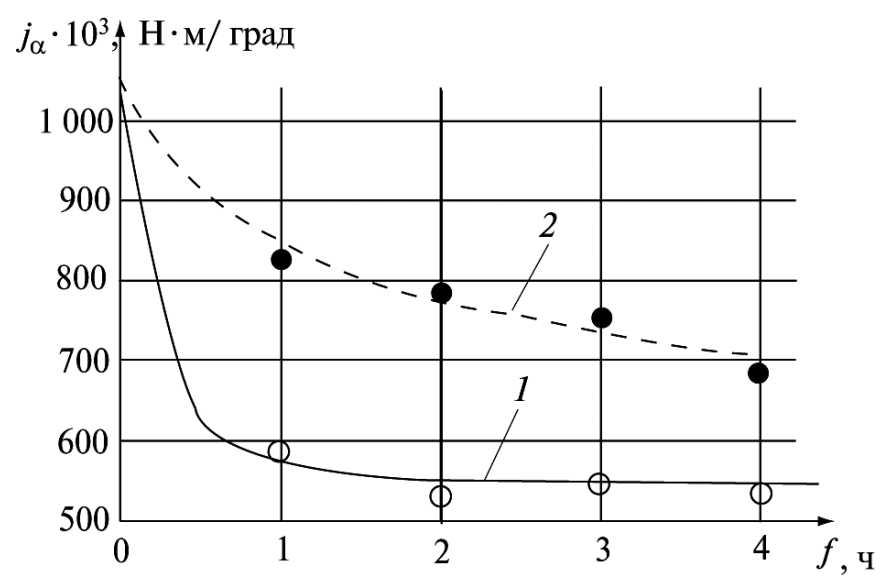

Puc. 1. Изменение угловой жесткости НСС в плоскости формирования точности обработки:

1 - конструкции в целом; 2 - конструкции при отключенном шпинделе 
этих параметров, необходимо выбрать систему отсчета, в которую входит система координат, хронометр для оценки параметров закона движения и линейно-угловые средства для измерения количественной оценки линейных и угловых параметров траектории.

Объектом изучения при токарной схеме обработки является шпиндель, движущийся под действием тепла относительно станины, по которой перемещается суппорт. Исследования показали, что ось шпинделя имеет пространственное движение и в данном случае ее положение однозначно характеризуется пятью степенями свободы (тремя линейными $-X, Y, Z$ и двумя угловыми $-\alpha$ и $\beta$ ). Реализация системы отсчета выражается в привязке системы координат к НСС. Примем за начало координат точку пересечения плоскости фланца патрона с осью шпинделя, а оси координат расположим, как показано на рис. 2.

В начало координат поместим часы и линейно-угловые средства измерения. Реализация системы отсчета в схематичном варианте представлена на рис. 3.

На фланце патрона закреплена труба 1 , служащая базой для отсчета положения оси всех пяти первичных преобразователей (два в вертикальной плоскости, два в горизонтальной и один вдоль оси 6). Первичные преобразователи расположены на кронштейне 4-8, который, в свою очередь, закреплен на станине 9 станка. Осевой датчик регистрирует движение шпинделя относительно станины. Для того чтобы исключить влияние длины станины сам датчик установлен на инваровом стержне 7 , база шпинделя реализована в виде пятки 3, закрепленной также на инваровом стержне 2, который зафиксирован на фланце патрона и служит началом отсчета при движении шпинделя в осевом направлении.

При исследовании жесткости НСС нагрузку прикладывали к патрону посредством динамометра камертонного типа. Деформации НСС при действии механической силы измеряли с помощью указанных первичных преобразователей. Эти же преобразователи оценивали параметры термического движения по всем пяти степеням свободы оси шпинделя.

Было исследовано поведение НСС при разных тепловых возмущениях, что обеспечива-

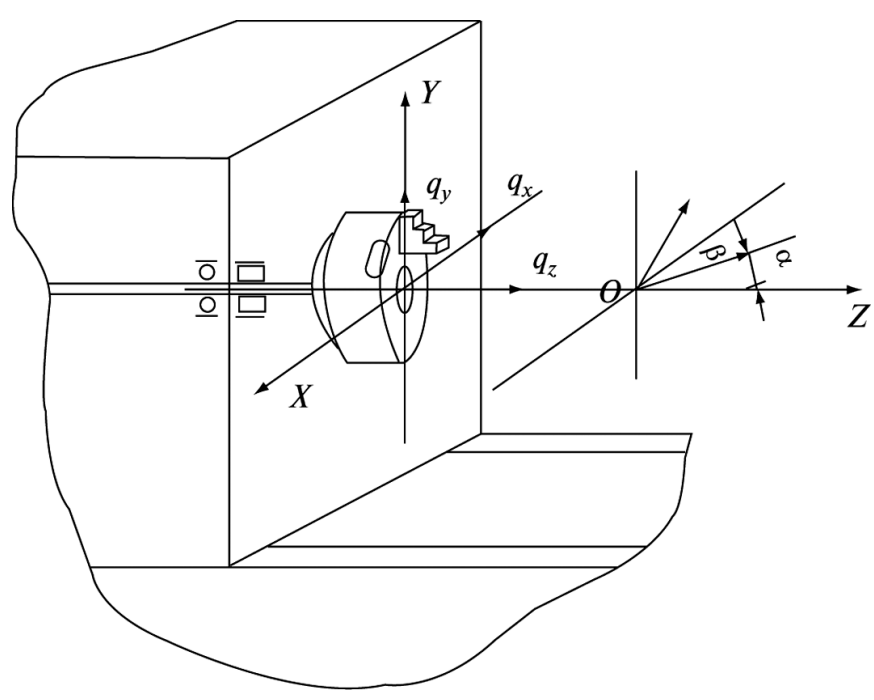

Puc. 2. Система координат для исследования параметров термического движения

лось различными частотами вращения шпинделя. В качестве примера представлена одна из реализаций при частоте вращения шпинделя 1 600 мин $^{-1}$ :

$$
\begin{aligned}
& j_{\alpha}(T)=1100-\left\{0,98 \alpha\left(T^{\prime}\right)-0,37 \beta\left(T^{\prime}\right) \cdot 10^{4}-\right. \\
& \left.-\left[2,32 q_{y}\left(T^{\prime}\right)-2,07 q_{z}\left(T^{\prime}\right)+0,014 q_{x}\left(T^{\prime}\right) \cdot 10\right]\right\} .
\end{aligned}
$$

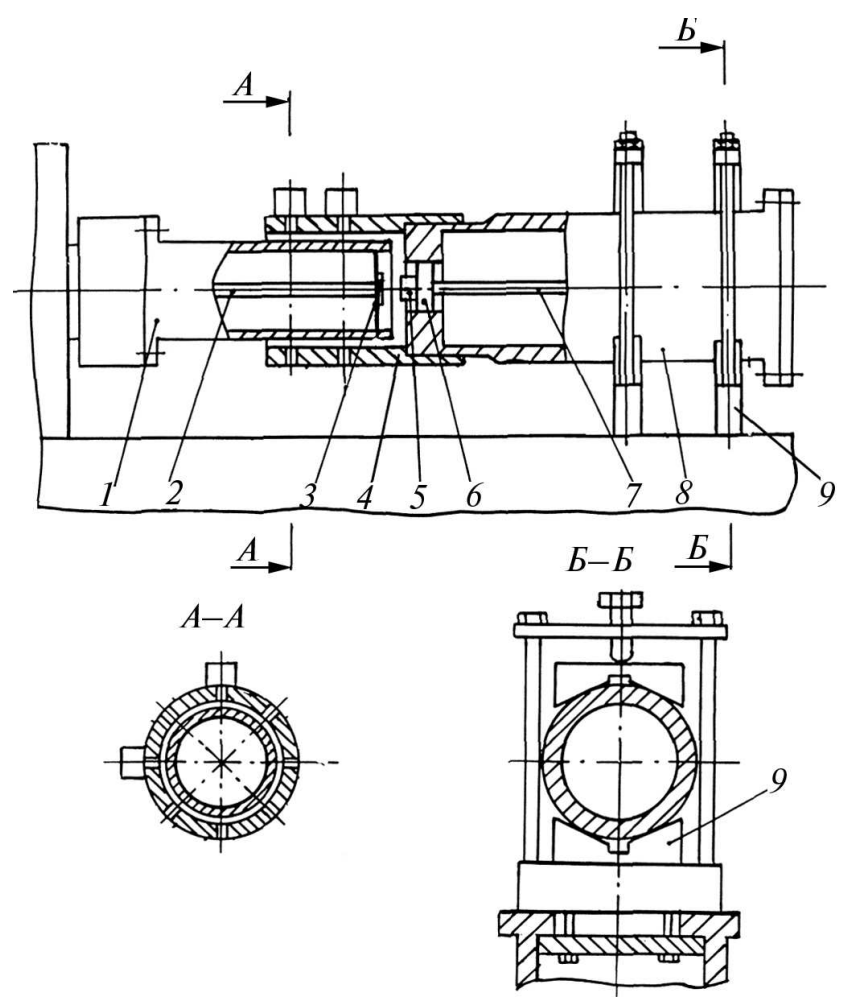

Рис. 3. Расположение измерительной системы на станке 
В общем виде изменение жесткости от времени работы в течение смены описывается выражением

$$
j_{\alpha}(T)=1100 \cdot \mathrm{e}^{-0,116 T^{\prime}},
$$

где 1100 - начальная жесткость, Н· м /рад; $T^{\prime}-$ время исследования, $T^{\prime}=6$ ч.

Из результатов эксперимента следует, что характер изменения жесткости НСС не противоречит характеру термических движений. По направлениям тепловых движений, согласно степеней свободы, участвуют как подвижные сопряжения, так и неподвижные стыки. В подвижных сопряжениях механизм движения основан на изменении размера деталей сопряжения, а в неподвижных стыках жесткость изменяется по другому закону. Из результатов, представленных в работе [2] следует, что жесткость стыка в значительной степени зависит от величины и конфигурации отклонения формы прилегающих поверхностей в стыке. Во время исследований, описанных в [2], изучалось влияние отклонения формы, возникающее во время изготовления деталей станка. При тепловых воздействиях отклонение формы обусловлено неравномерным прогревом конструкции и наличием градиента температур по конструкции.

Конструктивные особенности станка и его состояние в значительной степени определяют характер изменения жесткости под действием тепловых процессов, что обусловливает возникновение отклонения формы и расположения поверхностей произведенных деталей. Для того чтобы управлять этим процессом конструкторскими методами требуется дополнительная информация о характере поведения конструкции во время действия тепловых возмущений, которую можно получить, выполнив специальные исследования по данному направлению.

\section{Выводы}

1. Термические явления, протекающие во время рабочего процесса, способствуют возникновению термических движений конструкции, которые оказывают двойное действие на состояние конструкцию станка.

2. Изменение жесткости конструкции, способствующее образованию погрешности обработки, зависит от пространственного термического движения НСС.

3. Стабилизация геометрической точности конструкции является необходимым условием, но не достаточным для обеспечения точности станка.

4. Для стабилизации жесткости конструкции станка требуется стабилизация состояния его подвижных сопряжений и неподвижных стыков по всей конструкции.

\section{Литература}

1. Енджеевски E., Квасьны В. Влияние тепловых изменений зазора в подшипниках качения на жесткость шпиндельных узлов // Станки и инструменты. 1977. № 4. С. 10-12.

2. Левина З.М., Решетов Д.Н. Контактная жесткость машин. М.: Машиностроение, 1971. 264 с.

3. Точность механической обработки и пути ее повышения / Под. ред. А.П. Соколовского. М.-Л.: Машгиз, 1951. 487 с.

4. Базров Б.М. Технологические основы проектирования самонастраивающихся станков. М.: Машиностроение, 1974. $216 \mathrm{c}$.

\section{References}

1. Endzheevski E., Kvas'ny V. Vliianie teplovykh izmenenii zazora v podshipnikakh kacheniia na zhestkost' shpindel'nykh uzlov [The effect of thermal changes of the gap in rolling the stiffness spindles]. Stanki $i$ instrumenty [Tools and Instruments]. 1977 , no. 4, pp. $10-12$.

2. Levina Z.M., Reshetov D.N. Kontaktnaia zhestkost' mashin [Contact stiffness machines]. Moscow, Mashinostroenie publ., 1971. 264 p.

3. Tochnost' mekhanicheskoi obrabotki i puti ee povysheniia [Machining accuracy and ways to improve it]. Ed. Sokolovskii A.P. Moscow, Leningrad, Mashgiz publ., 1951. 487 p.

4. Bazrov B.M. Tekhnologicheskie osnovy proektirovaniia samonastraivaiushchikhsia stankov [Technological basis for the design of self-adjusting machine]. Moscow, Mashinostroenie publ., 1974. 216 p.

Статья поступила в редакцию 07.03.2013

\section{Информация об авторе}

ДМИТРИЕВ Борис Михайлович (Москва) - доктор технических наук, доцент кафедры «Металлообрабатывающие станки и комплексы». МГТУ им. Н.Э. Баумана (105005, Москва, Российская Федерация, ул. 2-я Бауманская, д. 5, стр. 1, е-таil: dmitriev@bmstu.ru).

DMITRIEV Boris Mikhailovich (Moscow) - Dr. Sc. (Eng.), Associate Professor of «Metalworking machinery» Department. Bauman Moscow State Technical University (BMSTU, building 1, 2-nd Baumanskaya str., 5, Moscow, Russian Federation, e-mail: dmitriev@bmstu.ru). 